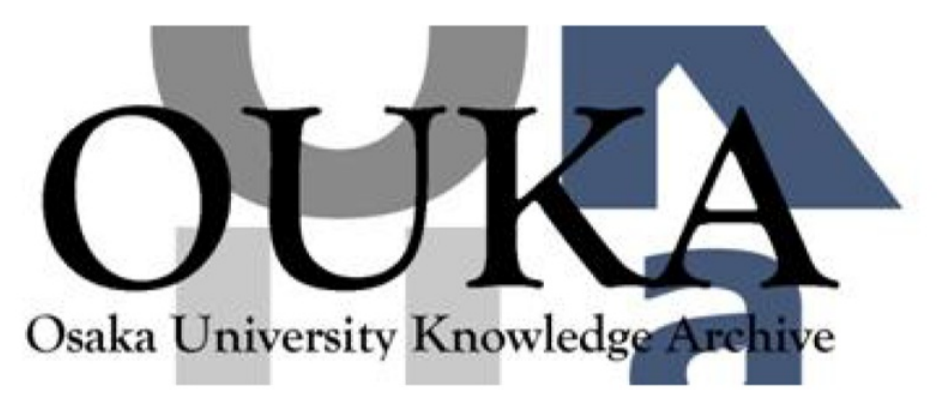

\begin{tabular}{|c|l|}
\hline Title & $\begin{array}{l}\text { Symmetric Potential Lattice and Smooth } \\
\text { Propagation of Tai L-Free Discrete Breathers }\end{array}$ \\
\hline Author(s) & Doi, Yusuke; Yoshimura, Kazuyuki \\
\hline Citation & Physical Review Letters. 117(1) p. 014101 \\
\hline Issue Date & $2016-06-30$ \\
\hline oaire:version & VoR \\
\hline URL & https://hdl. handle. net/11094/84550 \\
\hline rights & Copyright 2016 by the American Physical Society \\
\hline Note & \\
\hline
\end{tabular}

Osaka University Knowledge Archive : OUKA

https://ir. Library. osaka-u. ac. jp/

0saka University 


\title{
Symmetric Potential Lattice and Smooth Propagation of Tail-Free Discrete Breathers
}

\author{
Yusuke Doi \\ Department of Adaptive Machine Systems, Graduate School of Engineering, \\ Osaka University, 2-1 Yamadaoka, Suita, Osaka 565-0871, Japan \\ Kazuyuki Yoshimura \\ NTT Communication Science Laboratories, NTT Corporation 3-1 Morinosato, Wakamiya, Atsugi, Kanagawa 243-0198, Japan
}

(Received 15 October 2015; published 30 June 2016)

\begin{abstract}
We present a particular type of one-dimensional nonlinear lattice that supports smoothly propagating discrete breathers. The lattice is constructed by imposing a particular symmetry on its potential function. This symmetry crucially affects the profile and motion of a traveling discrete breather. We show that any traveling discrete breather is truly localized with no tail and can smoothly propagate with a constant velocity. Theoretical analysis using an average Lagrangian explains this numerical observation.
\end{abstract}

DOI: 10.1103/PhysRevLett.117.014101

Discrete breathers (DBs) are space-localized vibration modes that ubiquitously emerge in dynamical systems with both discreteness and nonlinearity. The concept of DB was introduced by Sievers and Takeno [1], and it has been of great interest $[2,3]$. Two types of DBs are known to be possible, i.e., stationary and traveling DBs. Long-lived traveling DBs have been found numerically [4-6]; they propagate along the lattices without noticeable decay for a long time. Such traveling DBs are of considerable interest from the viewpoint of energy transport in discrete dynamical systems, and their properties have been investigated in a variety of lattice models [7-10].

In general, the lattice discreteness tends to reduce the mobility of DB. For instance, an approximate traveling DB produced by kicking a stationary DB loses its velocity during its propagation, and it is eventually trapped at a certain lattice site in the Fermi-Pasta-Ulam (FPU) lattice [6]. This velocity loss is significant especially for strongly localized DBs, i.e., in the highly discrete regime, while it is insignificant for weakly localized DBs.

On the other hand, it is possible to precisely compute a traveling DB solution without velocity loss numerically in various lattices. The most remarkable feature of such a traveling DB is that it has a small amplitude tail extending over the lattice [7-10]. Another feature is that it does not propagate with a constant velocity but with periodically varying velocity, i.e., the nonsmooth propagation [8]. The period of this velocity variation is just the time needed for propagating one lattice space. It is known that both the tail and the velocity variation vanish in the continuum limit, where the DB is very weakly localized $[9,11]$. This fact indicates that the two features are just manifestations of the lattice discreteness effect.

A natural question is whether there exists any lattice model that allows constant-velocity traveling DBs with no tail, i.e., free from the discreteness effect, except for the integrable lattice [12]. If it exists, such a special lattice may offer high mobility in the sense that even an approximate traveling DB can propagate with little velocity loss. A related, even more fundamental, issue is to clarify an essential property of the lattice potential that causes the discreteness effect. Some special lattices of nonlinear Schrödinger type, which allow constant-velocity traveling DBs with no tail, have been constructed [13,14]. However, investigations on these lattices do not elucidate the above fundamental issue.

In this paper, we construct a particular FPU-type lattice, which has a potential function consisting of pairwise interactions and may be physically reasonable, and we numerically show that it allows constant-velocity traveling DBs with no tail and, moreover, exhibits a high mobility of approximate traveling DBs. The present lattice is an extension of the four-particle lattice constructed in Ref. [15] to an arbitrary degree of freedom, and it has a particular symmetry in its potential function. We show that the lack of this symmetry is the origin of the discreteness effect on propagation of DB.

Let us consider a class of one-dimensional lattices defined by the Hamiltonian

$$
H=\frac{1}{2} \sum_{n=1}^{N} p_{n}^{2}+\Phi\left(q_{1}, q_{2}, \ldots, q_{N}\right)
$$

where $p_{n}$ and $q_{n}$ are the momentum and the displacement of the $n$th particle, respectively, and $N$ is the number of particles. We assume the periodic boundary condition and that the potential $\Phi$ is invariant for cyclic permutations of the coordinates, i.e., $\Phi\left(q_{2}, q_{3}, \ldots, q_{N}, q_{1}\right)=$ $\Phi\left(q_{1}, q_{2}, \ldots, q_{N-1}, q_{N}\right)$.

We introduce the complex normal mode coordinates $U_{m} \in \mathbb{C}, m=-N / 2+1, \ldots, N / 2$ via the transformation

$q_{n}=\frac{(-1)^{n}}{\sqrt{N}} \sum_{m=-N / 2+1}^{N / 2} U_{m} \exp \left[i \theta_{m} n\right], \quad n=1, \ldots, N$, 
where $\theta_{m}=2 \pi m / N$ and $U_{-m}=\bar{U}_{m}$. The variable $U_{N / 2}=$ $\left(q_{1}+\cdots+q_{N}\right) / \sqrt{N}$ represents the center of mass. Let us define the map $\mathcal{T}_{\lambda}: \mathbb{C}^{N-1} \rightarrow \mathbb{C}^{N-1}$ by

$\mathcal{T}_{\lambda}: U_{m} \mapsto U_{m} \exp \left[-i \theta_{m} \lambda\right], \quad m=-N_{h}, \ldots, N_{h}$,

where $\lambda \in \mathbb{R}$ and $N_{h}=N / 2-1$. Given a displacement pattern $\mathbf{q}=\left(q_{1}, \ldots, q_{N}\right)$ satisfying $U_{N / 2}=\sum_{n=1}^{N} q_{n} / \sqrt{N}=0$, the action of $\mathcal{T}_{\lambda}$ with $\lambda=1$ corresponds to shifting $\mathbf{q}$ by one lattice site and reversing its sign in the configuration space, i.e., $q_{n} \mapsto-q_{n-1}$. Thus, $\mathcal{T}_{\lambda}$ with an arbitrary $\lambda$ is regarded as a continuous extension of this discrete shifting. Note that the action of $\mathcal{T}_{\lambda}$ is not defined for $m=N / 2$. This is because $U_{N / 2}$ has to be a real number, but the mapping of Eq. (3) would read $U_{N / 2} \mapsto U_{N / 2} \exp [-i \pi \lambda]$ and not be closed within $\mathbb{R}$ for $\lambda \notin \mathbb{Z}$.

In the coordinates (2), Hamiltonian (1) reads

$$
H=\frac{1}{2} \sum_{m=-N_{h}}^{N_{h}+1}\left|\dot{U}_{m}\right|^{2}+\Phi\left(\mathbf{U}, U_{N / 2}\right),
$$

where $\mathbf{U}=\left(U_{-N_{h}}, \ldots, U_{N_{h}}\right)$. We can decompose the potential as $\Phi\left(\mathbf{U}, U_{N / 2}\right)=\Phi(\mathbf{U}, 0)+\mathcal{R}\left(\mathbf{U}, U_{N / 2}\right)$, where $\mathcal{R} \equiv \Phi\left(\mathbf{U}, U_{N / 2}\right)-\Phi(\mathbf{U}, 0)$. The $U_{N / 2}$-independent part $\Phi(\mathbf{U}, 0)$ can be decomposed into two parts, $\Phi_{\mathrm{s}}(\mathbf{U})$ and $\Phi_{\mathrm{a}}(\mathbf{U})$, in such a way that $\Phi_{\mathrm{s}}(\mathbf{U})$ is invariant with respect to $\mathcal{T}_{\lambda}$; i.e., $\Phi_{\mathrm{s}}\left(\mathcal{T}_{\lambda} \mathbf{U}\right)=\Phi_{\mathrm{s}}(\mathbf{U})$ for any $\mathbf{U} \in \mathbb{C}^{N-1}$ and any $\lambda \in \mathbb{R}$, while $\Phi_{\mathrm{a}}(\mathbf{U}) \equiv \Phi(\mathbf{U}, 0)-\Phi_{\mathrm{s}}(\mathbf{U})$ is asymmetric with respect to $\mathcal{T}_{\lambda}$. We call the former part $\Phi_{\mathrm{s}}(\mathbf{U})$ the symmetric part. Performing this decomposition in Eq. (4), we have

$$
H=\frac{1}{2} \sum_{m=-N_{h}}^{N_{h}+1}\left|\dot{U}_{m}\right|^{2}+\Phi_{\mathrm{S}}(\mathbf{U})+\Psi\left(\mathbf{U}, U_{N / 2}\right),
$$

where $\Psi \equiv \Phi_{\mathrm{a}}(\mathbf{U})+\mathcal{R}\left(\mathbf{U}, U_{N / 2}\right)$ is regarded as the asymmetric part of the whole potential $\Phi\left(\mathbf{U}, U_{N / 2}\right)$.

Let $\mathcal{I}=\left\{(\mathbf{q}, \mathbf{p}) \in \mathbb{R}^{2 N} \mid \sum_{n=1}^{N} q_{n}=\sum_{n=1}^{N} p_{n}=0\right\}$. This is the subspace specified by $U_{N / 2}=\dot{U}_{N / 2}=0$. We call lattice (1) or (5) a symmetric lattice if $\mathcal{I}$ is its invariant subspace and $\Psi(\mathbf{U}, 0)=\Phi_{\mathrm{a}}(\mathbf{U})=0$. These conditions mean that the Hamiltonian subsystem restricted on $\mathcal{I}$ has a potential consisting of the symmetric part $\Phi_{\mathrm{s}}$ only.

We carry out an approximate analysis using the average Lagrangian method to investigate the propagation property of DBs in a symmetric lattice. Consider the lattice (5), and let $\tilde{\mathbf{U}}(t)=\left(\tilde{U}_{-N_{h}}(t), \ldots, \tilde{U}_{N_{h}+1}(t)\right)$ be a stationary DB solution of it, $\tilde{\mathbf{U}}(t)=\tilde{\mathbf{U}}(t+T)$, and $\omega_{\mathrm{DB}} \equiv 2 \pi / T$ is its internal frequency. To approximate a corresponding traveling DB, we assume the ansatz

$q_{n}(t)=\mu+\frac{(-1)^{n}}{\sqrt{N}} \sum_{m=-N_{h}}^{N_{h}} \tilde{U}_{m}(t) \exp \left[i \theta_{m}(n-x(t))\right]$, where $x(t)$ is the collective coordinate representing the position of the traveling DB and $\mu$ is the dc bias constant given by the time average of $\tilde{U}_{N / 2} / \sqrt{N}$. Using this ansatz, we have

$\dot{q}_{n}=\frac{(-1)^{n}}{\sqrt{N}} \sum_{m=-N_{h}}^{N_{h}}\left\{\dot{\tilde{U}}_{m}-i \theta_{m} \dot{x} \tilde{U}_{m}\right\} \exp \left[i \theta_{m}(n-x)\right]$.

Note that the ansatz (6) is equivalent to the substitution of $\mathcal{T}_{x} \tilde{\mathbf{U}}(t)$ and $\mu$ into Eq. (2) as $\left\{U_{m}\right\}_{m=-N_{h}}^{N_{h}}$ and $U_{N / 2}$, respectively. The variation of $x$ is assumed to be much slower than that of $\tilde{\mathbf{U}}(t)$.

If we substitute Eq. (7) into the kinetic energy $\mathcal{K}=\frac{1}{2} \sum_{n=1}^{N} \dot{q}_{n}^{2}$ and average it with respect to the fast variables $\tilde{\mathbf{U}}(t)$, then we obtain the average kinetic energy $\langle\mathcal{K}\rangle_{T}=\left\langle\sum_{m=-N_{h}}^{N_{h}}\left|\tilde{U}_{m}\right|^{2} / 2\right\rangle_{T}+\left\langle\sum_{m=1}^{N_{h}} \theta_{m}^{2}\left|\tilde{U}_{m}\right|^{2}\right\rangle_{T} \dot{x}^{2}$, where $\langle\cdot\rangle_{T}$ stands for the time average over one period $T$. We denote the first and second averages in $\langle\mathcal{K}\rangle_{T}$ with $C_{1}$ and $A$, respectively, since they do not depend on time but they are constants.

The averaged potential energy is obtained as $\langle\mathcal{V}\rangle_{T}=$ $\left\langle\Phi_{\mathrm{s}}\left(\mathcal{T}_{x} \tilde{\mathbf{U}}\right)\right\rangle_{T}+\left\langle\Psi\left(\mathcal{T}_{x} \tilde{\mathbf{U}}, \mu\right)\right\rangle_{T}=\left\langle\Phi_{\mathrm{s}}(\tilde{\mathbf{U}})\right\rangle_{T}+\left\langle\Psi\left(\mathcal{T}_{x} \tilde{\mathbf{U}}, \mu\right)\right\rangle_{T}$. The first term $\left\langle\Phi_{\mathrm{s}}(\tilde{\mathbf{U}})\right\rangle_{T}$ is a constant $C_{2}$ since it is independent of $x$. The second term $\left\langle\Psi\left(\mathcal{T}_{x} \tilde{\mathbf{U}}, \mu\right)\right\rangle_{T}$ is a function of $x$, which we denote with $W(x)$. The function $W(x)$ is a periodic function with period one (lattice spacing), i.e., $W(x)=W(x+1)$, because of the invariance of $\Phi$ for cyclic permutations of $\mathbf{q}$.

Combining $\langle\mathcal{K}\rangle_{T}$ and $\langle\mathcal{V}\rangle_{T}$, we obtain the averaged Lagrangian $\langle\mathcal{L}\rangle_{T}=\langle\mathcal{K}\rangle_{T}-\langle\mathcal{V}\rangle_{T}$ in the form

$$
\langle\mathcal{L}\rangle_{T}=A \dot{x}^{2}+C-W(x),
$$

where $C=C_{1}-C_{2}$. If the lattice is a symmetric one, $\langle\mathcal{L}\rangle_{T}$ has the form of a free-particle Lagrangian since $W(x)$ vanishes on $\mathcal{I}$. Then $\dot{x}(t)=$ const follows. This result of average Lagrangian analysis indicates that a traveling DB propagates smoothly with a constant velocity in a symmetric lattice. On the other hand, for nonsymmetric lattices such as the FPU lattice, Eq. (8) indicates that a traveling DB behaves like a particle in the periodic potential $W(x)$.

A simple method for obtaining a symmetric lattice is to consider an ordinary lattice model and remove the asymmetric part from its potential. However, this leads to an unphysical model. Here we construct a physically reasonable symmetric lattice. We look for such a lattice, assuming the potential in Eq. (1) of the form

$\Phi=\sum_{n=1}^{N}\left[\frac{1}{2}\left(q_{n+1}-q_{n}\right)^{2}+V\left(q_{n}\right)\right]+\sum_{r=1}^{N / 2} \sum_{n=1}^{N} \frac{b_{r}}{4}\left(q_{n+r}-q_{n}\right)^{4}$,

where $V(q)=\alpha q^{2} / 2+\beta q^{4} / 4$ is the on-site potential and $b_{r}, \alpha$, and $\beta$ are constants. This is a potential consisting of 
long-range pairwise interactions: $b_{r}$ represents the interaction strength with the $r$ th neighbor particle. The lattice with potential (9) may be regarded as a generalization of the FPU- $\beta$ lattice since it reduces to the FPU- $\beta$ lattice when $\alpha=\beta=0$ and $b_{r}=0$ for $r \geq 2$.

If we substitute Eq. (2) into Eq. (9), the potential $\Phi$ is rewritten in terms of $\mathbf{U}$ [16]. It can be shown that $\mathcal{I}$ is invariant if and only if $\beta=0$. When $\beta=0$ and $U_{N / 2}=0$, the rest of the asymmetric part is obtained as follows:

$\Phi_{\mathrm{a}}(\mathbf{U})=-\frac{4}{N} \sum_{\substack{i, j, k, l=-N_{h} \\ i+j+k+l= \pm N}}^{N_{h}} \psi^{(i, j, k, l)}(\mathbf{b}) U_{i} U_{j} U_{k} U_{l}$,

where $\mathbf{b}=\left(b_{1}, \ldots, b_{N / 2}\right)$. The coefficients $\psi^{(i, j, k, l)}(\mathbf{b})$ are linear combinations of $\left\{b_{r}\right\}_{r=1}^{N / 2}$, and they are given by

$$
\begin{aligned}
\psi^{(i, j, k, l)}(\mathbf{b})= & \sum_{q=1}^{N / 4}\left[b_{2 q-1} c_{(2 q-1) i} c_{(2 q-1) j} c_{(2 q-1) k} c_{(2 q-1) l}\right. \\
& \left.-b_{2 q} s_{2 q i} s_{2 q j} s_{2 q k} s_{2 q k}\right],
\end{aligned}
$$

where $c_{\alpha}=\cos (\alpha \pi / N)$ and $s_{\alpha}=\sin (\alpha \pi / N)$. Let $Q=$ $\left\{(i, j, k, l) \in \mathbb{Z}^{4} \mid-N_{h} \leq i, j, k, l \leq N_{h}, i+j+k+l= \pm N\right\}$. The lattice becomes symmetric if and only if the asymmetric part $\Phi_{\mathrm{a}}(\mathbf{U})$ vanishes. The condition $\Phi_{\mathrm{a}}(\mathbf{U})=0$ is equivalent to

$$
\psi^{(i, j, k, l)}(\mathbf{b})=0, \quad \forall(i, j, k, l) \in Q .
$$

This gives a set of linear equations for $\left\{b_{r}\right\}_{r=1}^{N / 2}$.

Let $S_{1}=\{(0, n+1, N / 2-n, N / 2-1) \mid 1 \leq n \leq N / 4-$ $1\} \subset Q \quad$ and $\quad S_{2}=\{(2-m, m, N / 2-1, N / 2-1) \mid m=1$ or $3 \leq m \leq N / 4+1\} \subset Q$. The equations $\psi^{(i, j, k, l)}(\mathbf{b})=$ $0,(i, j, k, l) \in S_{1} \cup S_{2}$ are $N / 2-1$ linearly independent equations, and they have a nontrivial solution $\mathbf{b} \neq 0$ since the number of equations is smaller than that of the unknowns. It can be shown that this nontrivial solution also solves the other equations in Eq. (12). The following theorem holds.

Theorem Given $N$, let $\mathbf{b}=\left(b_{1}, \ldots, b_{N / 2}\right)$ be a nontrivial solution of the equations $\psi^{(i, j, k, l)}(\mathbf{b})=0,(i, j, k, l) \in S_{1} \cup S_{2}$. Suppose $\beta=0$. Then Hamiltonian (1) with potential (9) is a symmetric lattice for any $\alpha \in \mathbb{R}$.

The solution $\mathbf{b}$ is uniquely determined up to a constant factor. Figure 1 shows numerically calculated $b_{r}$ for $b_{1}=1$. It is found that $b_{r} / b_{1}$ can be approximated by $r^{-2}$ for $r$ not close to $N / 2$. For $r \simeq N / 2, b_{r} / b_{1}$ converges to a certain nonzero value, which depends on $N$, due to a finite-size effect. We call the lattice constructed here the pairwise interaction symmetric lattice (PISL).

A numerically exact traveling DB solution can be calculated for a given pair of internal frequency $\omega_{\mathrm{DB}}$ and velocity $v_{\text {DB }}$ by using the Newton-Raphson method, provided that the velocity is given by $v_{\mathrm{DB}}=f / g$ (sites/periods) with $f$

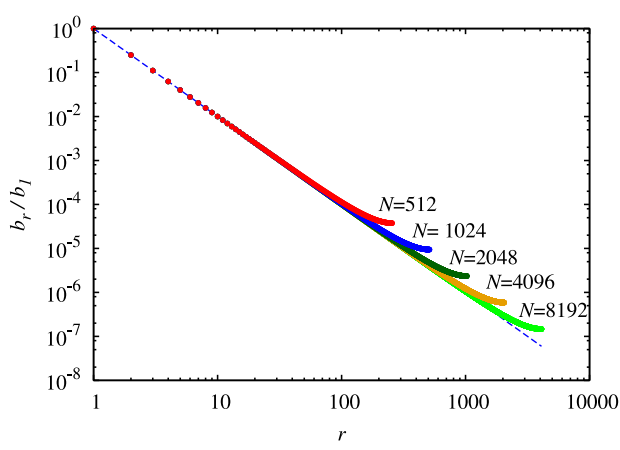

FIG. 1. Interaction strength $b_{r}$ obtained by solving Eq. (12) numerically $\left[2 b_{r}\right.$ is plotted for $r=N / 2$ since the interaction of a particle with its $N / 2$ th neighbor is summed twice in Eq. (9)]. The dashed line indicates the scaling law $b_{r} / b_{1}=r^{-2}$.

and $g$ integers; i.e., the DB propagates $f$ lattice sites during $g$ internal vibrations.

Figures 2(a)-2(d) show profiles of traveling and stationary DBs in terms of the site energy $e_{n}=V\left(q_{n}\right)+$ $\left[\left(q_{n+1}-q_{n}\right)^{2}+\left(q_{n}-q_{n-1}\right)^{2}\right] / 4+\sum_{r=1}^{N / 2} b_{r}\left[\left(q_{n+r}-q_{n}\right)^{4}+\right.$ $\left.\left(q_{n}-q_{n-r}\right)^{4}\right] / 8$, for two PISLs and two nonsymmetric lattices. As nonsymmetric lattices, we employ the FPU- $\beta$ lattice and the nonlinear on-site lattice that has $\alpha=\beta=1$ and $\left\{b_{r}\right\}_{r=1}^{N / 2}$ the same as the PISL. Figure 2(a) shows that the traveling DB has a profile that is quite different from that of the stationary DB in the FPU- $\beta$ lattice. The traveling DB has a spatially extended tail, where the site energy does not decrease with increasing distance from the DB center, but it is nearly constant; the stationary DB has no tail and exhibits an exponential decrease in the site energy. In contrast, as shown in Fig. 2(b) and 2(c), there is no significant difference in profile between the traveling and
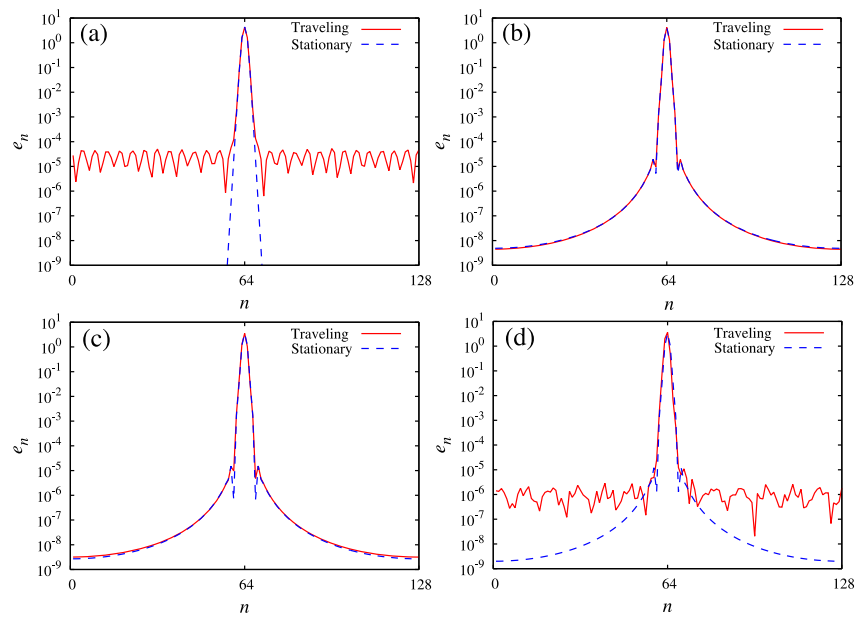

FIG. 2. Site energy for traveling (red solid line) and stationary DB (blue dashed line) in (a) the FPU- $\beta$ lattice, (b) PISL $(\alpha=\beta=0)$, (c) PISL with linear on-site $(\alpha=1, \beta=0)$, and (d) nonlinear on-site lattice $(\alpha=\beta=1)$. Parameters are $\omega_{\mathrm{DB}}=\pi$ and $v_{\mathrm{DB}}=1 / 10$. 

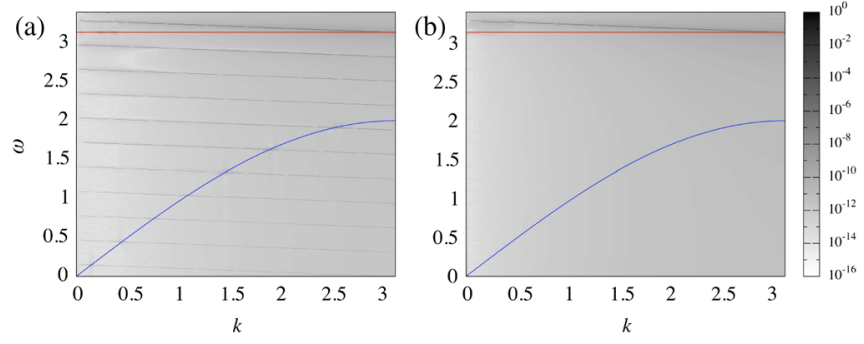

FIG. 3. Space-time Fourier spectrum of traveling DB for (a) FPU lattice and (b) PISL $(\alpha=\beta=0) .|S(k, \omega)|^{2}$ is presented by color in log scale for $\omega_{\mathrm{DB}}=\pi$ and $v_{\mathrm{DB}}=1 / 10$. The blue line indicates the dispersion curve. The red line indicates $\omega_{\mathrm{DB}}$.

stationary DBs in the PISLs for both $\alpha=0$ and 1. It should be noted that the travelings DBs have no constantamplitude tail. The on-site potential nonlinearity $(\beta \neq 0)$ destroys the potential symmetry. Consistently, the traveling DB has a spatially extended tail in Fig. 2(d) for the nonlinear on-site lattice.

The tail of a traveling DB in the FPU- $\beta$ lattice emerges due to resonances of the internal vibration of the $\mathrm{DB}$ with phonon modes [9]. This mechanism is general and has been pointed out also for the nonlinear Schrödinger lattice [10]. The tailfree profile of traveling DB in Fig. 2(b) suggests a lack of such resonances in the PISL. Figure 3 shows $|S(k, \omega)|^{2}$, where $S(k, \omega)=\int_{-\infty}^{\infty} U_{k}(t) e^{i \omega t} d t / \sqrt{2 \pi}$. In Fig. 3(a), multiple lines in $(k, \omega)$ space appear and intersect with the dispersion curve in the FPU- $\beta$ lattice. Each intersection corresponds to the resonance. In contrast, in Fig. 3(b), only a single line appears near its internal frequency, and this line does not intersect with the dispersion curve, showing that no resonance occurs in the PISL. The results in Figs. 2 and 3 show that emergence of tails of traveling DBs and the associated resonances are due to the asymmetric part $\Psi$.

Given a traveling DB, we define its center position by $x(t)=\sum_{n=1}^{N} n e_{n}(t)$, where $e_{n}$ is the site energy. We use a short-time average of $x$ given by $X(t)=\int_{t-\tau}^{t+\tau} x(s) d s / 2 \tau$ to investigate the propagation process of the traveling DB.

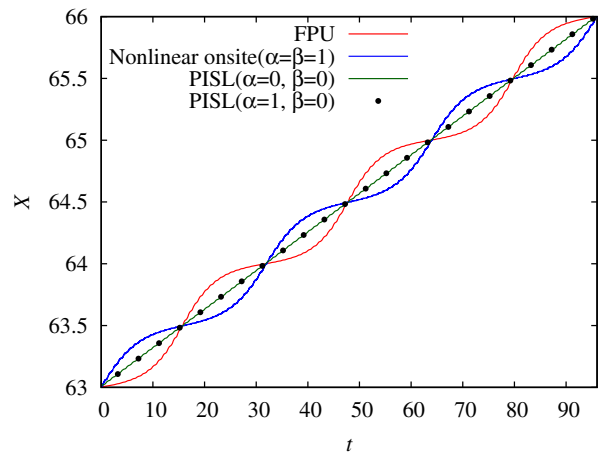

FIG. 4. The center $X$ of traveling DB vs $t$ for the FPU- $\beta$ lattice, PISLs $(\alpha=\beta=0)$ and $(\alpha=1, \beta=0)$, and a nonlinear on-site lattice. The averaged velocity $v_{\mathrm{DB}}=1 / 16$, and the internal frequency $\omega_{\mathrm{DB}}=\pi$.

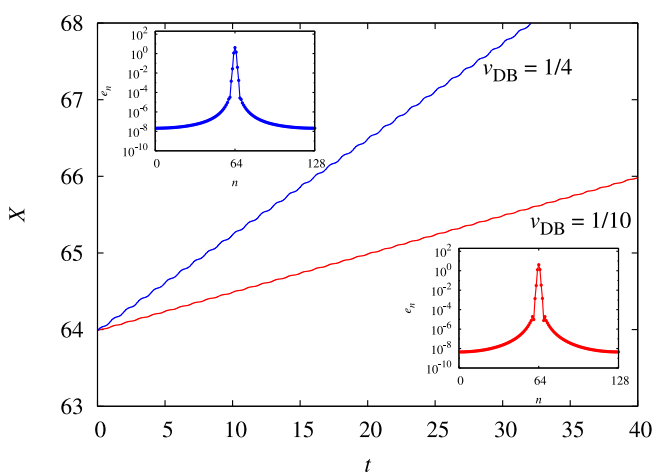

FIG. 5. The center $X$ of the traveling DB vs $t$ for PISL $(\alpha=\beta=0)$. Parameters are $v_{\mathrm{DB}}=1 / 4,1 / 10$ with $\omega_{\mathrm{DB}}=\pi$. The inset shows the DB profile.

The short-time average is performed in order to reduce fluctuations of $x(t)$ due to the fast internal DB vibration. The derivative $\dot{X}(t)$ may be regarded as the instantaneous velocity of the DB. Figure 4 shows $X(t)$ of traveling DBs with $\omega_{\mathrm{DB}}=\pi$ and $v_{\mathrm{DB}}=1 / 16$. In the FPU- $\beta$ lattice and the nonlinear on-site lattice, the DB propagates nonsmoothly with periodically varying velocity $\dot{X}(t): \dot{X}$ takes the maximum and minimum values when $X$ is at the midpoint between two lattice sites and at a lattice site, respectively, in the FPU- $\beta$ lattice; the opposite variation is observed in the nonlinear on-site lattice. In contrast, the DB propagates smoothly with a constant $\dot{X}(t)$ in each PISL. Examples of traveling DBs with different $v_{\mathrm{DB}}$ are shown in Fig. 5 for the PISL, demonstrating that DBs have no tail and that they propagate with constant velocities for various $\omega_{\mathrm{DB}}$ and $v_{\mathrm{DB}}$ values in PISL.

The smooth and nonsmooth propagation processes in the PISLs and the other lattices shown in Figs. 4 and 5 are consistent with the theoretical results of the average Lagrangian analysis. To further compare numerical and theoretical results, we show trajectories of numerically exact traveling DBs in $(X, \dot{X})$ space in Fig. 6 . In the case of the FPU- $\beta$ lattice, the trajectories behave just like the motion of a particle in a periodic potential in Fig. 6(a). Similar results for the FPU- $\beta$ lattice have already been reported [17]. On the other hand, the trajectories behave just like free-particle motion in the PISL, as in Fig. 6(b).
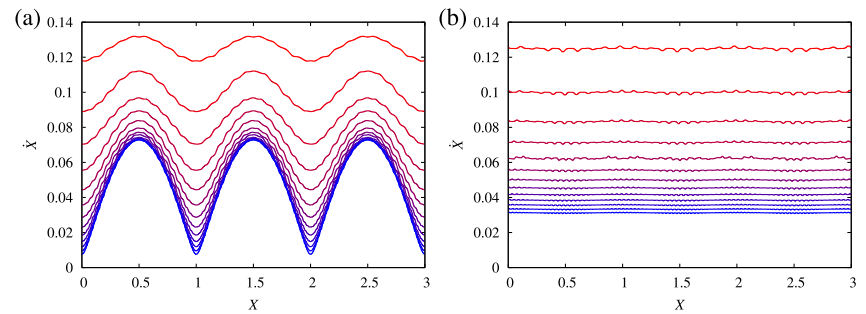

FIG. 6. Averaged trajectory of traveling DB in $(X, \dot{X})$ space with $\omega_{\mathrm{DB}}=\pi$ and $v_{\mathrm{DB}}=1 / g(g=4,5, \ldots, 16)$. (a) The FPU- $\beta$ lattice and (b) PISL $(\alpha=\beta=0)$. 

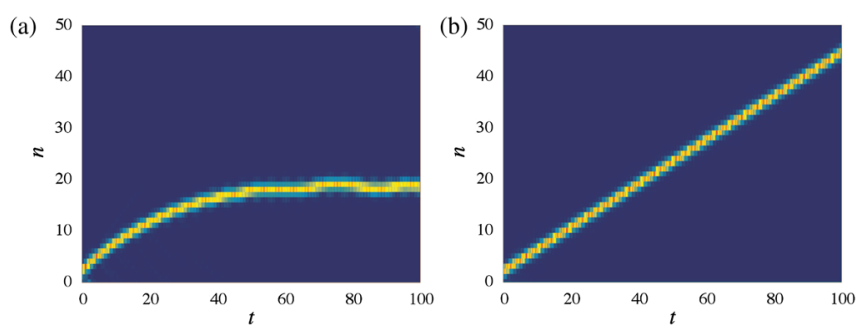

FIG. 7. Propagation of approximate traveling DB with $\omega_{\mathrm{DB}}=\pi$ and the resultant velocity $v_{\mathrm{DB}}=17 / 20$. Site energy is shown for (a) the FPU- $\beta$ lattice and (b) the PISL $(\alpha=\beta=0)$.

These behaviors in two lattices coincide with and validate the average Lagrangian analysis. Based on the numerical results in Figs. 4-6 and the theoretical analysis, we may conclude that the asymmetric part $\Psi$ of the potential causes the nonsmooth propagation of traveling DB.

Figure 7 shows spatiotemporal plots of the site energy of approximate traveling DBs, which are obtained by perturbing exact stationary DBs. In Fig. 7(a), an approximate DB loses its velocity gradually, and it is eventually trapped at a lattice site in the FPU- $\beta$ lattice. In contrast, in Fig. 7(b), even an approximate DB propagates smoothly without any noticeable velocity loss in the PISL. This confirms that a high mobility of approximate traveling DB is achieved in the PISL.

We have also considered an approximate PISL that has truncated interactions up to $M$ th $(M \ll N / 2)$ nearestneighbor particles and confirmed that it still supports high mobility of DB. Numerical results in the truncated PISL are shown in Ref. [16].

The first author (Y.D.) was partially supported by a Grant-in-Aid for Scientific Research (C), No. 16K05041 from Japan Society for the Promotion of Science (JSPS).

[1] A. J. Sievers and S. Takeno, Intrinsic Localized Modes in Anharmonic Crystals, Phys. Rev. Lett. 61, 970 (1988).

[2] S. Flach and A. V. Gorbach, Discrete breathers-Advances in theory and applications, Phys. Rep. 467, 1 (2008).

[3] K. Yoshimura, Y. Doi, and M. Kimura, Localized modes in nonlinear discrete systems, in Progress in Nanophotonics
III, edited by M. Ohtsu and T. Yatsui (Springer, New York, 2014), p. 119.

[4] V. M. Burlakov, S. A. Kiselev, and V. N. Pyrkov, Computer simulation of intrinsic localized modes in one-dimensional and two-dimensional anharmonic lattices, Phys. Rev. B 42, 4921 (1990).

[5] K. Hori and S. Takeno, Moving self-localized modes for the displacement field in a one-dimensional lattice system with quartic anharmonicity, J. Phys. Soc. Jpn. 61, 2186 (1992).

[6] K. W. Sandusky, J. B. Page, and K. E. Schmidt, Stability and motion of intrinsic localized modes in nonlinear periodic lattices, Phys. Rev. B 46, 6161 (1992).

[7] S. Aubry and T. Cretegny, Mobility and reactivity of discrete breathers, Physica D (Amsterdam) 119, 34 (1998).

[8] S. Aubry, Discrete breathers: Localization and transfer of energy in discrete Hamiltonian nonlinear systems, Physica D (Amsterdam) 216, 1 (2006).

[9] K. Yoshimura and Y. Doi, Moving discrete breathers in nonlinear lattice: Resonance and stability, Wave Motion 45, 83 (2007).

[10] J. Gómez-Gardeñes, F. Falo, and L. M. Floría, Mobile localization in nonlinear Schrödinger lattices, Phys. Lett. A 332, 213 (2004).

[11] B.-F. Feng, Y. Doi, and T. Kawahara, Quasi-continuum approximation for discrete breathers in Fermi-Pasta-Ulam atomic chains, J. Phys. Soc. Jpn. 73, 2100 (2004).

[12] M. J. Ablowitz, and J. F. Ladik, Nonlinear differentialdifference equations and Fourier analysis, J. Math. Phys. (N.Y.) 17, 1011 (1976).

[13] D. E. Pelinovsky, Translationally invariant nonlinear Schrödinger lattices, Nonlinearity 19, 2695 (2006).

[14] S. V. Dmitriev, P. G. Kevrekidis, N. Yoshikawa, and D. J. Frantzeskakis, Exact stationary solutions for the translationally invariant discrete nonlinear Schrödinger equations, J. Phys. A 40, 1727 (2007).

[15] Y. Doi and K. Yoshimura, Translational asymmetry controlled lattice and numerical method for moving discrete breather in four particle system, J. Phys. Soc. Jpn. 78, 034401 (2009).

[16] See Supplemental Material at http://link.aps.org/ supplemental/10.1103/PhysRevLett.117.014101 for the detail of the decomposition of potential Eq. (9).

[17] P. A. Houle, Phase plane of moving discrete breathers, Phys. Rev. E 56, 3657 (1997). 